\title{
Some remarks on p-blocks of finite groups
}

\author{
By Tetsuro Okuyama
}

(Received Oct. 4, 1977; Revised Feb. 21, 1980)

In this paper we are concerned with modular representations of finite groups. Let $G$ be a finite group and $p$ a fixed rational prime. Let $K$ be a complete $p$-adic field of characteristic 0 and $R$ the ring of $p$-local integers in $K$ with the principal maximal ideal $(\pi)$ and the residue class field $R=R /(\pi)$ of characteristic $p$. We assume throughout the paper that fields $K$ and $R$ are both splitting fields for all subgroups of the given group $G$. We mention here [2] and [3] as general references for the modular representation theory of finite groups.

1. In this section we shall give some necessary and sufficient condition for $G$ to be $p$-nilpotent. If $B$ is a $p$-block of $G$, then let $\operatorname{Irr}(B)$ denote the set of irreducible $K$-characters of $G$ in $B$. For a class function $\theta$ of $G$ we put $\theta_{B}=\sum_{\chi \in \operatorname{Irr}(B)}(\theta, \chi) \chi$. Let $B_{0}(G)$ denote the principal $p$-block of $G$.

We prove the following.

THEOREM 1. Let $H$ be a subgroup of $G$ which contains a Sylow p-subgroup $P$ of $G$. If $1_{H^{G} B_{0}(G)}(x)=1$ for any $p$-element $x \neq 1$ in $G$, then $H$ controls the fusion of elements of $P$.

To prove the theorem we use the following elementary lemma which follows from Brauer's Second Main Theorem.

Lemma. Let $\theta$ be a class function of $G, x$ a p-element of $G$ and $B$ a p-block of $G$. Then $\theta_{B}(x)=\sum \theta_{\mid C_{G}(x) b}(x)$ where $b$ ranges over the set of p-blocks of $C_{G}(x)$ with $b^{G}=B$.

Proof of Theorem 1. Let $x \neq 1$ be an element of $P, C=C_{G}(x), B=$ $B_{0}(G)$ and $b=B_{0}(C)$. By Mackey decomposition we have $1_{H}{ }^{G} \mid C=\sum\left(1_{H^{y} \cap C}\right)^{C}$ where $y$ ranges over a complete set of representatives of $(H, C)$-double cosets in $G$. Thus the above lemma and the result of Brauer (Theorem 65.4 [2]) show that $1_{H B}{ }^{c} B(x)=\sum\left(1_{H^{y} \cap C}\right)^{c}{ }_{b}(x)$. If $x \in H^{y} \cap C$, then $\left(1_{H^{y} \cap C}\right)^{C}{ }_{b}(x)=\left(1_{H^{y} \cap C}\right)^{c}{ }_{b}$ (1). and if $x \notin H^{y} \cap C$, then $\left(1_{H^{y} \cap C}\right)^{C}{ }_{b}(x)=0$ by (6.3) IV in [3]. As $1_{H^{G}}{ }^{G}(x)=1$ by our assumption, $x \in H^{y} \cap C$ if and only if $y \in H C$. Therefore if $x^{y} \in H$ for some element $y$, then there exists an element $h$ in $H$ such that $x^{y}=x^{h}$ and therefore the theorem is proved.

As an easy corollary of Theorem 1 we have the following. 
Corollary 2. Let $P$ be a Sylow p-subgroup of $G$. Then $G$ is $p$ nilpotent if and only if $1_{P}{ }^{G} B_{0}(G)(x)=1$ for any p-element $x \neq 1$ of $G$.

Proof. If $G$ is $p$-nilpotent, then it is easy to show that $1_{P}{ }^{G} B_{0}(G)=1_{G}$ and therefore $1_{P}{ }^{G} B_{0}(G)(x)=1$ for any element $x$ of $G$. Conversely assume that $1_{P}{ }^{G} B_{0}(G)=1$ on $p$-elements $\neq 1$ of $G$. Then Theorem 1 shows that two elements of $P$ are conjugate in $G$ if and only if in $P$. Thus the corollary follows from the well-known result on Transfer Theory.

2. If $B$ is a $p$-block of $G$ then for an $R[G]$-module $V$ we define $V_{B}=$ $V e$ where $e$ is the centrally primitive idempotent of $R[G]$ corresponding to $B$ (in this paper modules will always be right unital). Let $L_{0}(G)$ denote the trivial $R$-free $R[G]$-module of $R$-rank 1 . If $V$ is an $R[G]$-module then let $\bar{V}=V / V(\pi)$ which is an $\bar{R}[G]$-module.

Let $P$ be a Sylow $p$-subgroup of $G$. If $P \triangleleft G$, then $L_{0}(P)^{G}$ is completely reducible and every irreducible $R[G]$-module has a vertex $P$. In this connection we have the following.

THEOREM 3. Let $G$ be a finite group and $B$ a p-block of $G$ with defect group $D$. Let $N=N_{G}(D)$ and $b$ a p-block of $N$ with $b^{G}=B$. then the following are equivalent.

(1) $G \triangleright D \operatorname{Ker} B$ where $\operatorname{Ker} B=\bigcap_{x \epsilon_{9} \operatorname{Irr}(B)} \operatorname{ker} \chi$.

(2) For every irreducible $R[G]$-module $L$ in $B L_{N}$ is also irreducible.

(3) $\overline{L_{0}(D){ }^{G}}{ }_{B}$ is completely reducible and every irreducible $R[G]$-module in $B$ has a vertex $D$.

Proof. $\quad(1) \rightarrow(3)$. Since $G \triangleright D \operatorname{Ker} B, \bar{L}_{0}(D)^{G}{ }_{B}$ is considered as an $\bar{R}[G / D]$ module. Every irreducible $R[G]$-module in $B$ has kernel containing $D$ and is projective as an $R[G / D]$-module. Thus the result follows.

$(3) \rightarrow(2)$. By our assumprion $L_{0}(D)^{G}{ }_{B}=\sum \oplus n_{i} L_{i}$ where $L_{i}^{\prime} s$ are irreducible $\bar{R}[G]$-modules in $B$. By Nakayama Relation (see [3], p 141) $n_{i}=$ $\operatorname{dim}_{\bar{R}} U_{i} /|D|$ where $U_{i}$ is the principal indecomposable $\bar{R}[G]$-module corresponding to $L_{i}$. Then assertion $(1) \rightarrow(3)$ shows that $\overline{L_{0}(D)^{N}{ }_{b}}=\sum \oplus m_{j} M_{j}$ where $M_{j^{\prime}} s$ are irreducible $\bar{R}[N]$-modules in $b$. By the same reason as the above $m_{j}=\operatorname{dim}_{\bar{R}} V_{j}|| D \mid$ where $V_{j}$ is the principal indecomposable $R[N]$ module corresponding to $M_{j}$. Then by Green Correspondence with respect to $(G, D, N)$ the numbers of $L_{i^{\prime}} s$ and $M_{j^{\prime}} s$ are equal and after suitable rearrangement $n_{i}=m_{i}$ and $L_{i}$ corresponds to $M_{i}$. Then by Nakayama Relation $U_{i N} \cong V_{i}$ and therefore $L_{i N}=M_{i}$.

$(2) \rightarrow(1)$. This is proved by the similar argument in [4] (Theorem 4). By our assumption $\cap \operatorname{Ker} L \supseteq D \operatorname{Ker} B$ where $L$ ranges over the set of all irreducible $\bar{R}[G]$-modules in $B$. Thus $D \operatorname{Ker} B=\cap \operatorname{Ker} L \triangleleft G$. 
REMARK 1. The equivalence of (1) and (2) in case $B=B_{0}(G)$ is the result of Isaacs and Smith (Theorem 4, [4]).

As a corollary of this theorem we have the following.

Corollary 4. Let $G$ be a finite group and $P$ a Sylow p-subgroup of $G$. Then $G$ has p-length 1 if and only if $\bar{L}_{0}(P)^{G}{ }_{B_{0}(G)}$ is completely reducible and every irreducible $\bar{R}[G]$-module in $B_{0}(G)$ has a vertex $P$.

REMARK 2. The condition that every irreducible $\bar{R}[G]$-module in $B_{0}(G)$ in the above can not be dropped as the group $S_{4}$, the symmetric group of degree 4 shows.

3. In this section we shall prove some results related to the result of Brauer (Theorem 2, [1]). In [1] Brauer has proved the following.

Theorem (Brauer, [1]). Let $G$ be a finite group and $P$ a Sylow $p$ subgroup of $G$. If $B$ is a p-block of $G$ with defect group $D \subseteq P$, then $\operatorname{dim}_{\bar{R}} \bar{L}_{0}(P)^{G}{ }_{B}=|P: D| v$, where $(p, v)=1$.

If $\operatorname{dim}_{\bar{R}} L_{0}(P)^{G}{ }_{B}$ is a power of $p$, then Brauer's Theorem implies that $B$ has the unique irreducible $\bar{R}[G]$-module in it. In particular, $G$ is $p$ nilpotent if and only if $\operatorname{dim}_{\bar{R}} \bar{L}_{0}(P)^{G} B_{0}(G)$ is a power of $p$. This is the result of Brauer (Corollary 2, [1]). Furthermore we have the following.

Corollary 5. Let $G, P, B$ and $D$ be as in the above. Assume furthermore $P \triangleright D$. If $\operatorname{dim}_{\bar{R}} \bar{L}_{0}(P)^{G}{ }_{B}$ is a power of $p$, then $G \triangleright D \operatorname{Ker} B$ and $[G, D] \subseteq[P, D] \operatorname{Ker} B$.

Proof. Let $N=N_{G}(D)$. By the theorem of Brauer $\bar{L}_{0}(P)^{a}{ }_{B}=L$ is the unique irreducible $\bar{R}[G]$-module in $B$ and has dimension $|P: D|$. Since $N \supseteq P, L_{N}$ is irreducible and therefore $G \triangleright D \operatorname{Ker} B$ by Theorem 3. In order to prove the second statement we may assume $\operatorname{Ker} B=1$ and $D \triangleleft G$. Let $V$ be an arbitrary $R$-free $R[D]$-module of $R$-rank 1 with $\operatorname{Ker} V \supseteq[P, D]$. Let $H$ be the inertia group of $V$ in $G$. By ([3], p 163) $V_{B \mid D}^{G}=n \sum \oplus V^{x}$ for some positive integer $n$ where $x$ ranges over a complete set of representatives of right $H$-cosets in $G$. Since $\bar{V}=\overline{L_{0}(D)}, \operatorname{dim}_{\bar{R}} \bar{V}^{G}{ }_{B}$ is a power of $p$ and therefore so is $|G: H|$. As $\operatorname{Ker} V \supseteq[P, D]$ it follows that $H \supseteq P$ and we have $G=H$. Thus $x^{-1} x^{y} \in \operatorname{Ker} V$ for elements $x \in D$ and $y \in G$ and therefore the result follows. 


\section{References}

[1] R. BRAUer: Notes on representations of finite groups I, J. London Math. Soc. (2), 13 (1976), 162-166.

[2] L. DoRnhofF: Group Representation Theory, Marcel Dekker, New York (1972).

[3] W. FEIT: Representations of Finite Groups, Yale University (1969).

[4] I. M. IsAacs and S. D. Smith: Anote on groups of p-length 1, J. Alg. 38 (1976), 531-535.

Osaka City University 